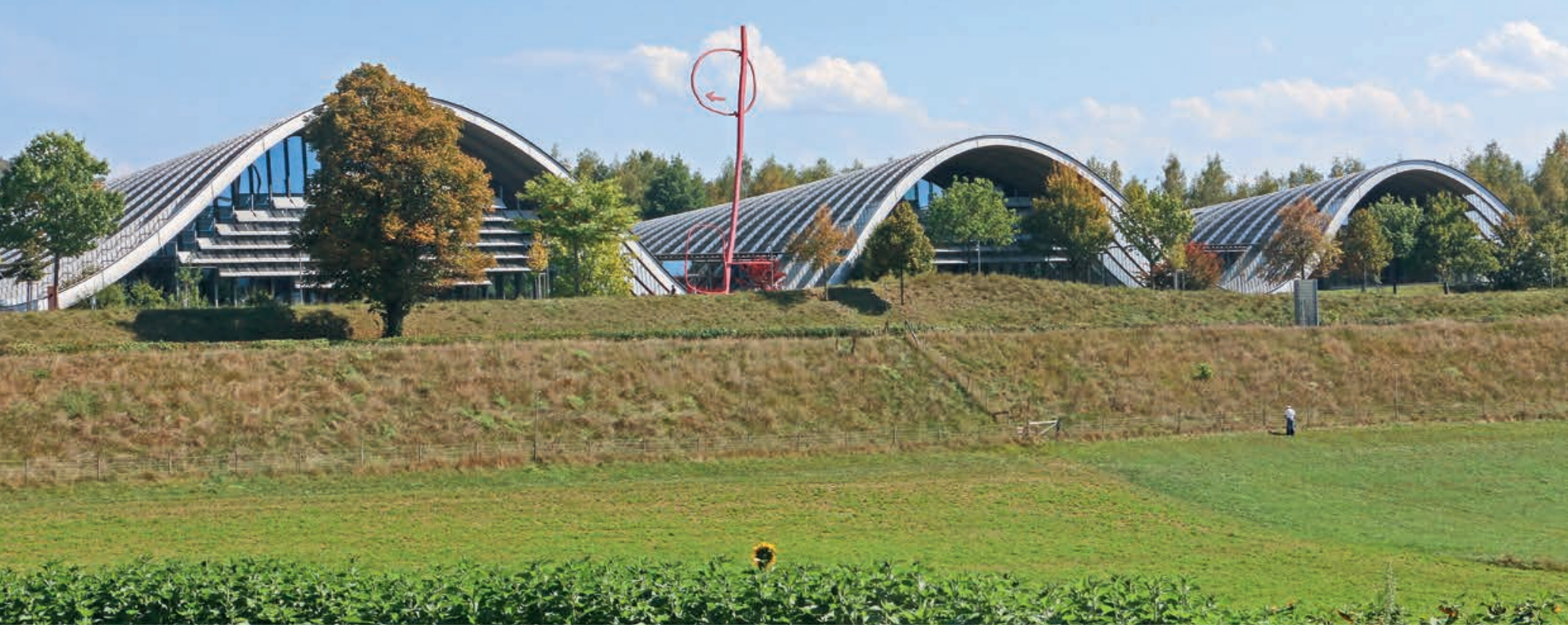

Das Zentrum Paul Klee in Bern war zum zweiten Mal Schauplatz des MedEd Symposiums.

\title{
Perspektiven der ärztlichen Bildung im 21. Jahrhundert
}

\section{Bruno Kesseli}

Dr. med. et lic. phil., Chefredaktor

Auch die fünfte Auflage des MedEd Symposiums, das am 12. September im Zentrum Paul Klee in Bern stattfand, bot dem Publikum ein interessantes Programm und reichlich Gelegenheit für einen intensiven Austausch zu Fragen der ärztlichen Bildung. Referenten aus dem In- und Ausland präsentierten spannende Erkenntnisse zum aktuellen Stand und den Perspektiven der Aus-, Weiter- und Fortbildung.

Werner Bauer, gastgebender Präsident des Schweizerischen Instituts für ärztliche Weiter- und Fortbildung SIWF, konnte sich in seiner Eröffnungsansprache über ein «kleines Jubiläum» freuen. Das von ihm initiierte MedEd Symposium erlebte in diesem Jahr bereits seine fünfte Auflage und hat sich bestens in der hiesigen Kongresslandschaft etabliert. Für den Präsidenten kein Grund, sich zurückzulehnen: «Wir müssen aufpassen, dass wir nun nicht in Routine verfallen», mahnte er sich selbst und sein Team.

Vorerst scheint diese Befürchtung unbegründet. Denn auch das diesjährige Programm überzeugte durch qualitativ gute Vorträge zu unterschiedlichen Themen der ärztlichen Bildung, setzte aber auch gezielt Schwer- punkte. Und ein guter Schuss Routine schadet ohnehin nicht, ganz im Gegenteil. Zur geschätzten - und gemäss Werner Bauer immer wieder verlangten - Routine gehört es, dass an den MedEd Symposien auch Sir William Osler Stammgast ist. Diesmal war der kanadische «Übervater» der Medizin unter anderem mit einem Zitat vertreten, das für einen Grossteil des Publikums aus Altersgründen vielleicht etwas provokativ war:

"Men above forty are rarely pioneers, rarely the creators in science or in literature [...] Once across this line we teachers and consultants are in constant need of postgraduate study as an antidote against premature senility. Daily contacts with the bright young minds of our assistants are important aids.»

(Lancet, 1900) 


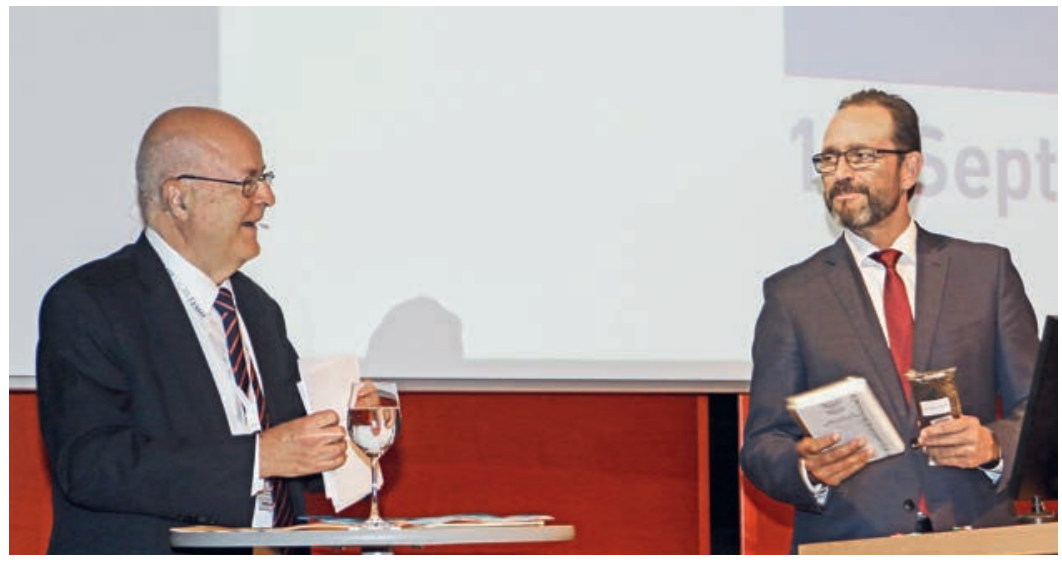

Gastgeber Werner Bauer und BAG-Direktor Pascal Strupler eröffneten das Symposium.

\section{Freundliche Worte des BAG-Direktors}

Ebenfalls zu den MedEd-Traditionen zählt mittlerweile das Kurzreferat von Pascal Strupler zum Auftakt des Symposiums, das «routinemässig» durch ein kleines Vorgeplänkel zwischen dem Direktor des Bundesamts für Gesundheit (BAG) und Werner Bauer eingeleitet wird. Diesmal bezog es sich auf "freundliche Post», die das SIWF gelegentlich aus dem BAG erhält. Dieses Attribut traf auch auf den Vortrag von Pascal Strupler zu. Der BAG-Direktor dankte den Weiterbildenden für ihr Engagement. Er unterstrich, dass die Aus- und Weiterbildung ein wichtiger Teil des schweizerischen Gesundheitssystems sei, das bekanntlich eine sehr gute Qualität aufweise. Ein Hauch von Kritik ging an diejenigen Vertreter der älteren Ärztegeneration, die als Ideal für Ärztinnen und Ärzte nach wie vor ausschliesslich den Weg sehen, sich «ein Leben lang kompromisslos der Medizin zu verschreiben", wie Strupler sinngemäss aus einem Zeitungsartikel zitierte. Die junge Ärztegeneration habe andere Bedürfnisse. «Darauf sollten wir uns einlassen und nicht dagegen ankämpfen.» Eine Qualität, die die Jungen unbestreitbar mitbrächten, bestehe darin, dass sie offen gegenüber neuen Methoden wie dem Simulationstraining seien.

\section{Die Vorzüge der Simulation}

Eben um diese Methode ging es in den ersten Hauptreferaten [1] des Tages. Oscar Traynor, Professor am Royal College of Surgeons of Ireland und Director of the National Surgical Training Centre, sprach zum Thema «Training with Simulation». Zwar befänden wir uns bekanntlich mittlerweile im 21. Jahrhundert, so Traynor, aber das chirurgische Training sei methodisch im 19. Jahrhundert steckengeblieben. Dies gelte eigentlich für alle medizinischen Disziplinen, während andere Bereiche, etwa die Luftfahrt und das Militär, aus naheliegenden Gründen vorwiegend mit Simulationstrainings arbeiteten. So seien Astronauten der NASA bereits bei ihrer ersten Mission technisch und situativ dank Simulationstraining bestens vorbereitet und voll trainiert. Dagegen würden

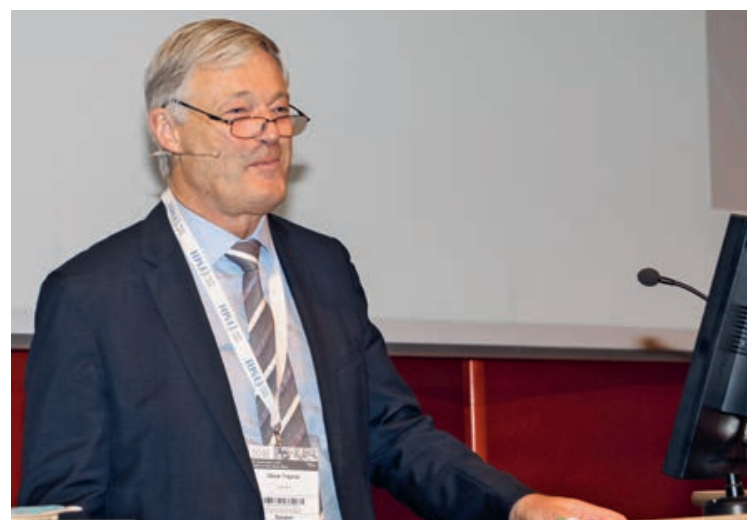

Oscar Traynor sieht die Simulation als Trainingsmethode des 21. Jahrhunderts.

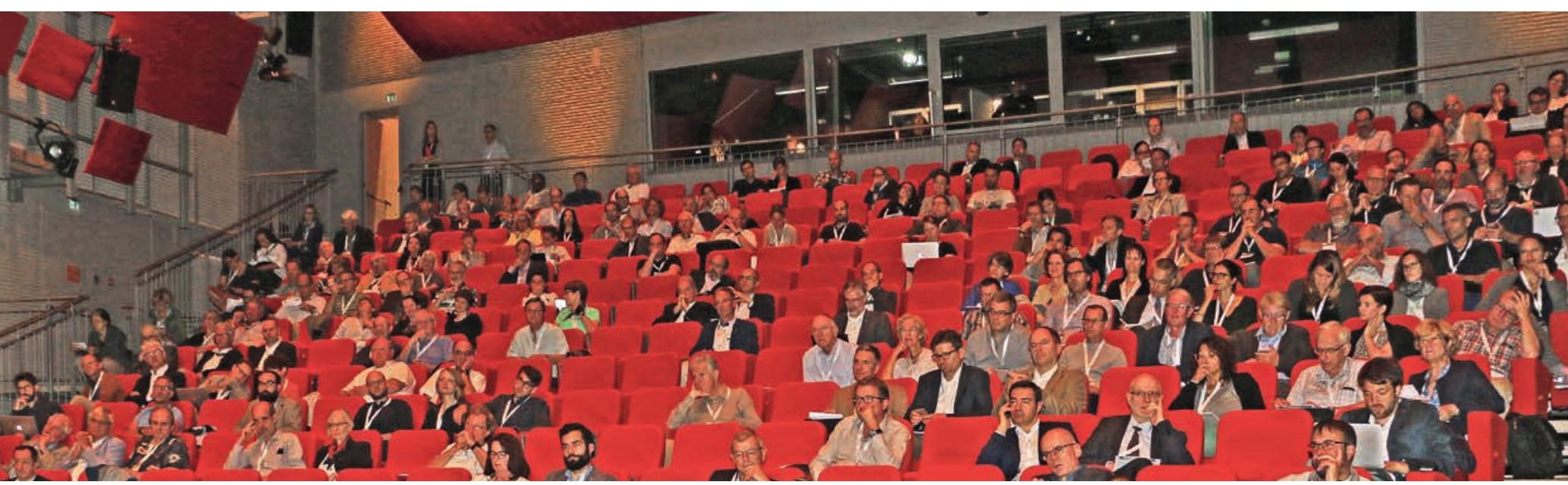

Auch in der 5. Auflage zog das MedEd Symposium ein grosses Publikum an. 


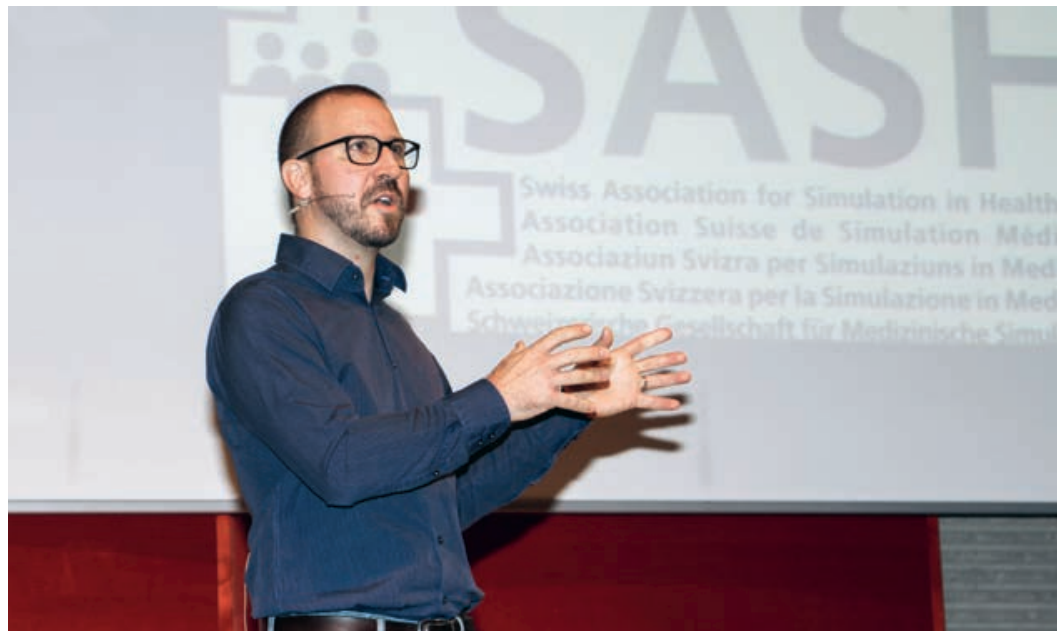

Adrian Marty, Leiter des Simulationszentrums des UniversitätsSpitals Zürich.

sich angehende Chirurgen gelegentlich bereits Expertenstatus zuschreiben, wenn sie eine bestimmte Operation ein einziges Mal durchgeführt hätten.

Traynor stellte den Wert einer herkömmlichen chirurgischen «Lehre» nicht in Abrede. Die nötigen Fertigkeiten seien auf diese Weise aber nur über viele Jahre zu erwerben, und die zunehmende Arbeitszeitbeschränkung stelle ein grosses Hindernis auf diesem Weg dar. Zudem werde die Medizin immer technischer, was die Komplexität teilweise erhöhe. Unter der Voraussetzung, dass sie in ein strukturiertes Curriculum eingebettet sei, könne die chirurgische Simulation gute Lösungen für viele aktuelle und künftige Herausforderungen bieten, mit denen die chirurgische Aus- und Weiterbildung konfrontiert sei. Traynor ging so weit, die Simulation als «die Trainingsmethode des 21. Jahrhunderts» zu bezeichnen. In seiner Präsentation zeigte er eindrücklich, dass sein Heimatland auf diesem Gebiet bereits einen hohen Standard erreicht hat.

Auch wenn man in der Schweiz noch nicht auf diesem

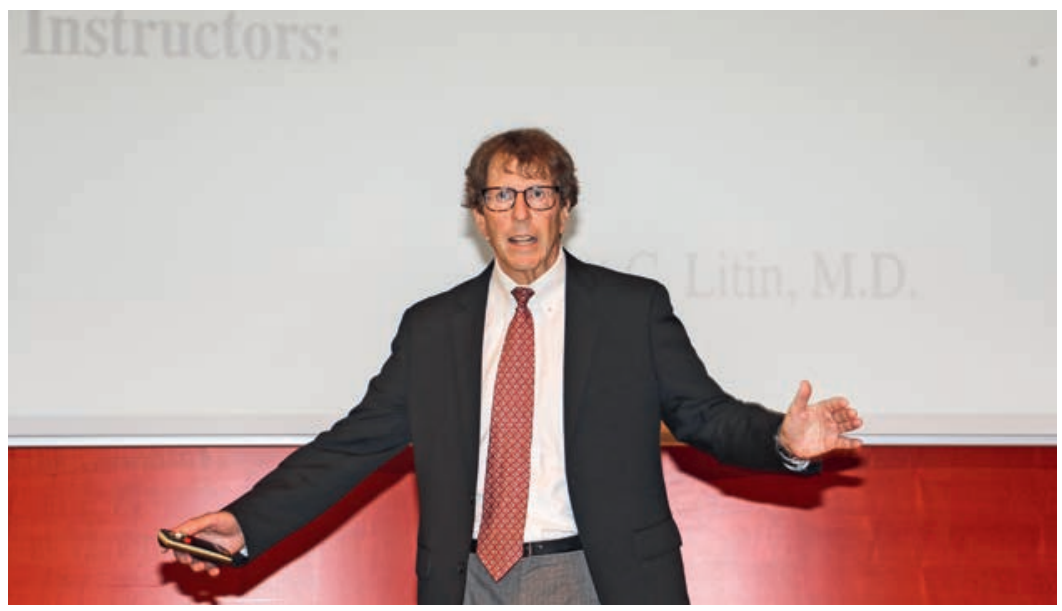

Vortrag als performance: Scott Litin.
Niveau ist, gibt es auch hierzulande vielversprechende Ansätze. Einer der Pioniere des Simulationstrainings ist Adrian Marty. Der Facharzt für Anästhesiologie leitet das Simulationszentrum des UniversitätsSpitals Zürich. In seinem Vortrag erläuterte er dem Publikum den Stellenwert des Simulationstrainings in der interprofessionellen Weiterbildung. Zwar «üben wir immer noch am liebsten am Patienten", wie Marty feststellte. Die Einsicht, dass Simulationstrainings gerade im interprofessionellen Kontext wertvoll und effizient seien, habe aber zugenommen, wenngleich er nach wie vor auch Ablehnung erlebe. Selbst bei Profiteams sei es erstaunlich, wie wenig die Teammitglieder teilweise über die Kompetenzen ihrer Kolleginnen und Kollegen wüssten. Der Kommunikation komme in der interprofessionellen Teamarbeit eine Schlüsselrolle zu, weil die Kompetenzen zur Lösung der meisten Probleme in der Regel im Team vorhanden seien. Martys Zentrum bietet deshalb nicht nur «technische» Trainings an, sondern auch Übungsszenarien zu interaktiven Situationen, beispielsweise für Teams aus Hausarztpraxen.

\section{Expertentipps für bessere Präsentationen}

Welche kommunikativen Prinzipien Vortragende beherzigen sollten, demonstrierte Scott C. Litin in seinem Referat unter dem Titel Clinical Teaching - more than just Presentation. Die Tipps des Past President der in Rochester, USA, beheimateten Mayo Clinic Faculty waren einfach, konkret und sehr überzeugend. Ein Vortrag sollte eine performance sein, so eine seiner Kernbotschaften. Um eine Botschaft ans Publikum zu bringen, ist die Art und Weise, in der sie vermittelt wird, genauso wichtig wie der transportierte Inhalt. Zentral sind eine entspannte Haltung, der Blickkontakt zum Publikum, eine lebendige Intonation und eine gemessene Sprechweise mit geschickt eingestreuten Pausen. Ein gelegentliches Lächeln und Humor können einen Vortrag wesentlich aufwerten. Eine gut organisierte Präsentation umfasst eine kurze Einführung, konzentriert sich auf wenige Punkte pro Folie und endet mit einer Zusammenfassung von maximal drei Punkten. Litin scheute nicht davor zurück, auf Details wie Schriftgrösse auf den Folien (er empfiehlt 32 Punkte als Standard, im Minimum 24 Punkte), Schrifttypen (keine Schriften mit Serifen) und Farbkombinationen (ideal ist weisse Schrift auf blauem Hintergrund) einzugehen. Fotos und kurze Videosequenzen können ebenfalls zum Erfolg einer Präsentation beitragen, nicht zuletzt, wenn sie die Aussagen der vortragenden Person in origineller oder witziger Weise ergänzen. Litin lieferte mit seinem Auftritt einen starken Beleg dafür, dass seine Methode funktioniert. 


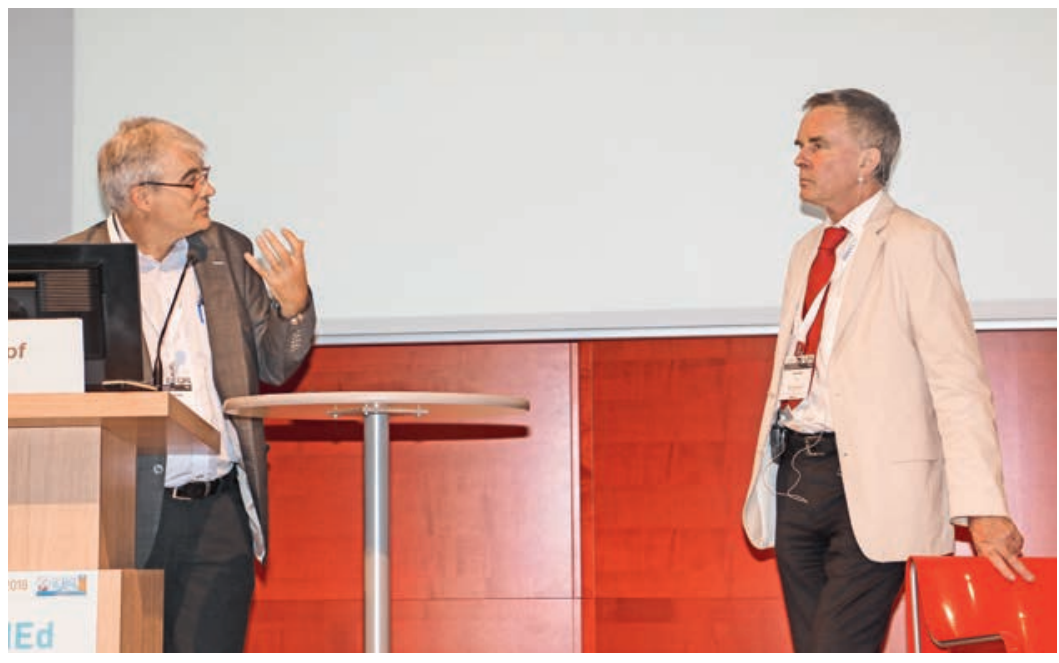

Prof. Mathieu Nendaz, Chairperson des Nachmittagsteils, im Gespräch mit Reto Krapf.

\section{Evidence-based Medicine (EBM) - Nutzen und Limitationen}

Das Hauptreferat des Nachmittags befasste sich mit dem Thema «Nutzen und Limitationen von EBM - Konsequenzen für die Fortbildung». Referent Reto Krapf stellte sein Licht zunächst etwas unter den Scheffel, indem er betonte, kein Fachspezialist der medizinischen (Fort-)Bildung zu sein. In der Folge präsentierte er dann aber fast schon ein Feuerwerk an facts and figures, die seine Konklusionen überzeugend belegten. Er stellte fest, dass die Evidence-based Medicine bei gut definierten Einzelerkrankungen "riesige Fortschritte» gebracht habe, zeigte sich aber überzeugt, dass sie für die «modernen, heterogenen, 'multimorbiden' Patientenpopulationen" ungenügend sei. Dies unter anderem deshalb, weil die Erkenntnisse der EBM nicht an denselben Patientenkollektiven gewonnen werden, die später eine auf diesen Erkenntnissen basierende Medikation oder Intervention erhalten. Ein grosses Potential ortet Reto Krapf bei der sogenannten BlockchainTechnologie. Darunter ist die Arbeit mit zentralen Datenbanken für den transparenten medizinischen Datenaustausch zwischen Patienten und den involvierten medizinischen «Partnern» zu verstehen. Auf diese Weise könnte es gelingen, die EBM durch eine MBE, eine Medicine-based Evidence, abzulösen, von der sich Krapf für den individuellen Patienten Vorteile verspricht. Voraussetzung für das Gelingen dieses Prozesses ist nach seiner Einschätzung allerdings das Primat einer guten ärztlichen Analyse und Betreuung.

\section{Drei Workshops und eine Podiums- diskussion}

Der Symposiumsnachmittag war zuvor durch drei parallel geführte Workshops eingeleitet worden [1], in denen die folgenden Themen behandelt und diskutiert wurden:

- «Supporting your learners: yes, but how?» [2]

- Ist interprofessionelle Weiterbildung machbar? [3]

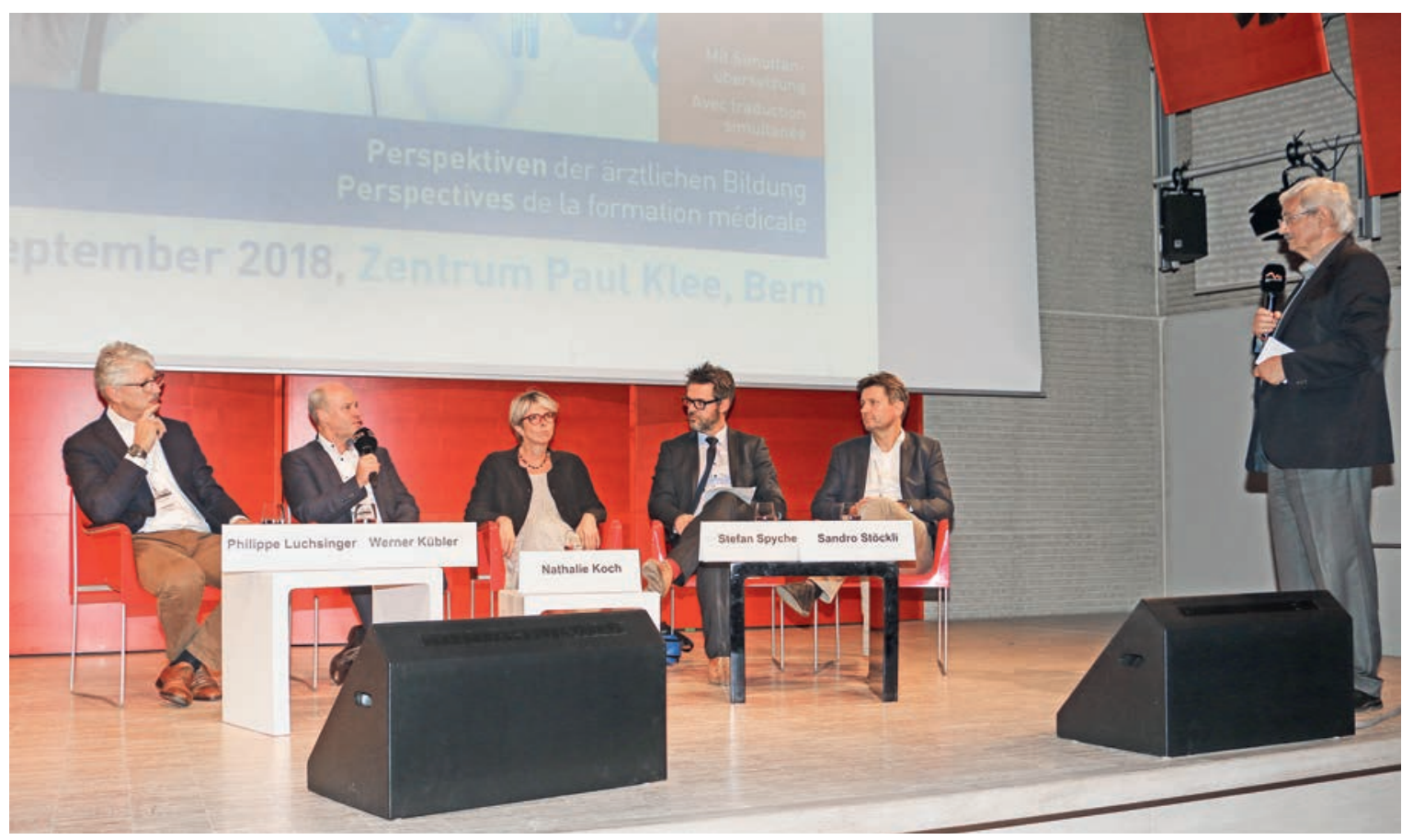

Das abschliessende Podium (von links): Philippe Luchsinger, Werner Kübler, Nathalie Koch, Stefan Spycher, Sandro Stöckli und Iwan Rickenbacher (Moderation). 
- Gestion du professionnalisme ou de son manquement en formation pré- ou postgraduée [4].

Den Ausklang des Symposiums bildete schliesslich eine von Iwan Rickenbacher in gewohnter Manier souverän moderierte Podiumsdiskussion [5]. Das Thema «Von der Fortbildung zur (Re-)Zertifizierung?» enthielt einigen potentiellen Zündstoff. Doch in der sehr konstruktiven Diskussion wurden keine «Bomben» gezündet. «Unsere Haltungen sind problemorientiert», hielt BAG-Vizedirektor Stefan Spycher gleich zu Beginn auf die Frage fest, ob unser Fortbildungssystem auf einem genügend hohen Niveau sei. Bevor eventuelle Massnahmen zu ergreifen seien, müsse man zuerst wissen, ob überhaupt ein Problem bestehe. Das Wissen in diesem Bereich sei aber noch so lückenhaft, dass es zu früh sei, bereits in die Details zu gehen. Philippe Luchsinger, Präsident von mfe Haus- und Kinderärzte Schweiz, zeigte sich mit dem aktuellen System zufrieden, da es ihm erlaube, seine fachlichen Defizite eigenverantwortlich zu beheben. Werner Kübler, Nathalie Koch und Sandro Stöckli orteten Verbesserungspotential bei der Strukturierung und Kontrolle der Fortbildung.

Die (Re-)Zertifizierung von Facharzttiteln scheint dagegen vorerst kein Thema zu sein - jedenfalls war sie es auf dem Podium nicht. Nathalie Koch sieht ein zentrales Problem bei der Fortbildungskontrolle im Umstand, dass die Medizin immer individueller werde. Nicht jeder müsse alles wissen, und deshalb werde es zunehmend schwierig, zu entscheiden, was zu prüfen sei. Eine Lanze für die Interprofessionalität brach Philippe Luchsinger. Den Hausärzten sei bewusst, dass sie ihren Job nicht weiter wie bisher stemmen könnten, konstatierte er. mfe engagiere sich deshalb stark für Interprofessionalität, die auch vermehrt in die Fortbildung einfliessen müsse. Werner Küblers Schlussvotum, dass in der Fortbildung weiterhin Raum sein sollte für Kreativität und Wettbewerb, schienen sich alle Podiumsgäste anschliessen zu können - dies dürfte auch auf den Grossteil des Publikums zutreffen. Das nächste MedEd Symposium findet am 11. September 2019 statt, wiederum im Zentrum Paul Klee, Bern.
SIWF Award 2018: Die Ausgezeichneten

Im Rahmen des 5. MedEd Symposiums wurden auch die Trägerinnen und Träger des SIWF Award 2018 für besonderes Engagement in der Weiterbildung bekanntgegeben. Diese Auszeichnung wird aufgrund der Nomination durch ehemalige Assistenzärztinnen und -ärzte vergeben. Folgende Weiterbildende erhielten in diesem Jahr eine Auszeichnung:

Dr Alfredo Addeo, Genève; PD Dr. med. Nicolas Attigah, Zürich; Dr Nadia Berkane, Genève; Dr. med. Hansulrich Blunier, Schüpfen; Dr. med. Christian Candrian, Lugano; Dr. med. Andrea Cantisani, Bern; Dr Christophe Coste, Chêne-Bourg; Dr Stephan Eliez, Genève; Dr. med. Katrin Feller, Bern; Dr. med. Aikaterini Galimanis, Bern; Dr. med. Andreas Geiser, Schlieren; Prof. Daniel Genné, Bienne; Dr. med. Katharina Gessler, Barmelweid; Dr Vincent Guggi, Payerne; Dr. med. Samuel Henz, Rorschach; Dr. med. Christine Roten, Bern; Dr. med. Christoph Schlegel, Luzern; Dr. med. Moritz Schürch, Glarus; Dr. med. Igor Schwegler, Zürich; Dr. med. Rahel Schwotzer, Zürich; PD Dr. med. Michael Soyka, Zürich; Dr. med. Victor Speidel, Thun; Dr. med. Gerd Stuckmann, Winterthur; Dr. med. BrigittaThomann, Schlieren; Dr. med. Seline Voney, Biel; Dr. med. Franca Wagner, Bern; Prof. Dr. med. Markus Weber, St. Gallen.

\section{Bildnachweis}

Fotos Bruno Kesseli

\section{Referenzen}

1 Die Präsentationen zu den Hauptreferaten und Seminaren sind zugänglich unter:www.fmh.ch/bildung-siwf/themen/mededsymposium.html

2 Leitung: Dr. med. Werner Bauer; Prof. Oscar Traynor, MD; Prof. Scott C. Litin.

3 Leitung: Dr. med. Raphael Stolz, Vizepräsident des SIWF; Dr. med. Dieter von Ow, Stv. Chefarzt, Zentrale Notfallaufnahme, Kantonsspital St. Gallen; PD Dr. med. André Mihaljevic, M.Sc., Klinik für Allgemein-, Viszeral- und Transplantationschirurgie, Universitätsklinikum Heidelberg.

4 Leitung: Nadia Bajwa, MD, MHPE Université de Genève, Faculté de Médecine, Hôpitaux Universitaires de Genève; Naïke Bochatay, MSc, Université de Genève, Faculté de Médecine; Aurélie Mauerhofer, MD, Centre Hospitalier Universitaire Vaudois; Anja Zyska Cherix, MD, Présidente de l'ASMAC-VSAOe.

5 Auf dem Podium diskutierten: Prof. Dr. Iwan Rickenbacher (Moderation), Honorarprofessor für politische Kommunikation der Universität Bern; Dre Nathalie Koch, Adjointe à la Direction médicale, responsable du suivi et du développement des compétences médicales CHUV, Lausanne; Dr. med. Werner Kübler, Spitaldirektor Universitätsspital, Basel; Dr. med. Philippe Luchsinger, Präsident Hausärzte Schweiz; Dr. Stefan Spycher, Vizedirektor, Leiter Direkti onsbereich Gesundheitspolitik, Bundesamt für Gesundheit (BAG); Prof. Dr. med. Sandro Stöckli, Mitglied der Geschäftsleitung Kantonsspital St. Gallen, Chefarzt Hals-Nasen-Ohrenklinik. 